\title{
Study on the Structure of Defects in a-Si:H Films by Positron Annihilation and Micro-Raman Spectroscopy
}

\author{
P.M. Gordo ${ }^{a, *}$, M.F. Ferreira Marques ${ }^{a, b}$, A.P. De Lima ${ }^{a}$ \\ AND Zs. KAJCSOS ${ }^{c}$ \\ ${ }^{a}$ ICEMS, Department of Physics, University of Coimbra \\ P-3004-516 Coimbra, Portugal \\ ${ }^{b}$ Depart. Eng. Quimica, Instituto Superior Engenharia \\ P-3031-199 Coimbra, Portugal \\ ${ }^{c}$ KFKI Res. Inst. for Nucl. and Part. Physics \\ P.O. Box 49, H-1525 Budapest 114, Hungary
}

\begin{abstract}
Thin films of hydrogenated amorphous silicon deposited on glass and crystalline silicon substrates by rf plasma enhanced chemical vapor deposition at different rf power were studied using slow positron beam and the Raman scattering spectroscopy in order to verify the influence of that deposition parameter on the film defect structure and on the degree of disorder. By positron annihilation spectroscopy, it was found that there are mainly two types of defects in the films: large vacancy clusters or voids and small vacancy type defects. By micro-Raman spectroscopy it was observed that the degree of structural disorder is lower for the film with large vacancy clusters and this finding was related to structural relaxation process. Light soaking induced changes attributed to major atomic rearrangements were also observed.
\end{abstract}

PACS numbers: 78.66.Jg, 78.70.Bj, 78.30.Ly

\section{Introduction}

Hydrogenated amorphous silicon (a-Si:H) is a technologically important material for semiconductor thin film production [1]. A wide variety of techniques are used for depositing these films, the rf plasma enhanced chemical vapor deposition (PECVD) with silane gas $\left(\mathrm{SiH}_{4}\right)$ being the most widely used technique for industrial production of good quality films. The amorphous silicon structure

*corresponding author; e-mail: pgordo@ci.uc.pt 
is, generally, characterized by the density of dangling bonds and by the standard deviation of the bond angle of the network and varies with the preparation technique [2]. The dangling bonds are most likely associated with small multivacancy complexes (divacancies, trivacancies, etc.) within the disordered tetrahedrally coordinated network. The positron technique, being highly sensitive to vacancy type defects, provides a useful contribution to the identification and understanding of the correlations between the film growth conditions and the optoelectronic properties of the film. Raman spectroscopy is a convenient technique to measure bond angle and dihedral angle disorder, since it is well established that an increase in the short-range disorder (bond angle deviation) is manifested by an increase in the line width of the transverse optic (TO) band. This relation was first observed by Beeman et al. [3] and is expressed by the equation: $\Gamma_{\mathrm{TO}}=15+6 \Delta \theta$, where $\Gamma_{\mathrm{TO}}$ $\left(\mathrm{cm}^{-1}\right)$ is the full width half maximum of the TO-like band and $\Delta \theta\left(^{\circ}\right)$ represents the root mean square (rms) bond angle deviation. Similarly, the shift to lower frequencies of the peak position of the TO spectral band, $\omega_{\mathrm{TO}}$, is associated with an increase in the average bond-length when compared to crystalline silicon (c-Si). As it is well known, the incident light induces degradation of the hydrogenated amorphous silicon electronic properties; this is the so-called Staebler-Wronsky (SW) effect. A theoretical interpretation [4] of the SW effect implies a decrease in the short-range order upon light soaking, i.e., an increase in $\Gamma_{\mathrm{TO}}$, which is related to the atomic rearrangement.

\section{Experimental}

The a-Si:H films were deposited on crystalline silicon (c-Si) and alkali free glass substrates by magnetically confined rf (13.56 MHz) PECVD technique, according to the optimized deposition conditions described here: substrate temperature, $T_{\mathrm{S}}=250^{\circ} \mathrm{C}$; deposition pressure, $200 \mathrm{mTorr}$; $\mathrm{SiH}_{4}$ flow, $F_{\mathrm{SiH}_{4}}=20 \mathrm{sccm}$ and magnetic field intensity, $B=1.6 \times 10^{-2} \mathrm{~T}$. The thickness of the films was approximately $500-600 \mathrm{~nm}$. The rf power, $P_{\mathrm{D}}$, was varied between 5 and $40 \mathrm{~W}$. Doppler broadening measurements, using a positron beam of $\approx 4 \mathrm{~mm}$ diameter with incident energies in the range from 0.01 to $30 \mathrm{keV}$, were performed on a-Si:H thin films deposited on c-Si substrate. Details of the experimental setup have been reported elsewhere [5]. The Raman measurements were performed in the backscattering geometry using a Raman microprobe (Olympus) with a 100 objective attached to a triple spectrometer (Jobin-Yvon, model TC 64000), configured in the subtractive mode, and a liquid-nitrogen-cooled CCD detector (ISA Instruments). For the present measurements the $514.5 \mathrm{~nm}$ laser line, obtained from an Ar-ion laser (Coherent Innova 300), was used. The power of the incident light was varied from $\approx 1.5 \mathrm{~mW}$ up to $\approx 2.5 \mathrm{~mW}$ with a beam diameter of $\approx 10 \mu \mathrm{m}$. During each measurement the power of the incident laser light was kept constant. All measurements were performed at room temperature. 


\section{Results and discussion}

The $S(E)$ and $W(E)$ data were calculated using a window of energy between (510.23-511.77) keV for the parameter $S$ and windows of energy between (503.70$509.27)$ and (512.73-518.30) keV for the $W$ parameter, respectively. The Doppler parameters, $S_{\text {film }}$ and $W_{\text {film }}$, of each a-Si:H films deposited on c-Si were obtained fitting the $S(E)$ and $W(E)$ data using the VEPFIT code [6], assuming samples consisting of two layers, one being the a-Si:H film and the c-Si substrate the other (with characteristic values of $S_{\mathrm{c}-\mathrm{Si}}=0.5102(3)$ and $W_{\mathrm{c}-\mathrm{Si}}=0.1111(9)$ ). Figure $1 \mathrm{a}$ shows the $S_{\text {film }}$ parameter normalized to $S_{\mathrm{c}-\mathrm{Si}}$ for various a-Si:H films deposited at different $\mathrm{rf}$ powers together with the positron diffusion length. The normalized $S_{\text {film }}$ parameter reaches a maximum already at a relatively low rf power value and decreases above this then steadily. On the other hand, the linear behavior of the $S_{\text {film }}-W_{\text {film }}$ plot (Fig. 1b) strongly suggests that in these films two types of open volume defects are present, leading to competing trapping at a nearsaturation level. The relative concentration of those trapping sites is dependent on the rf power deposition. The experimental boundary values of the $S_{\text {film }}$ are
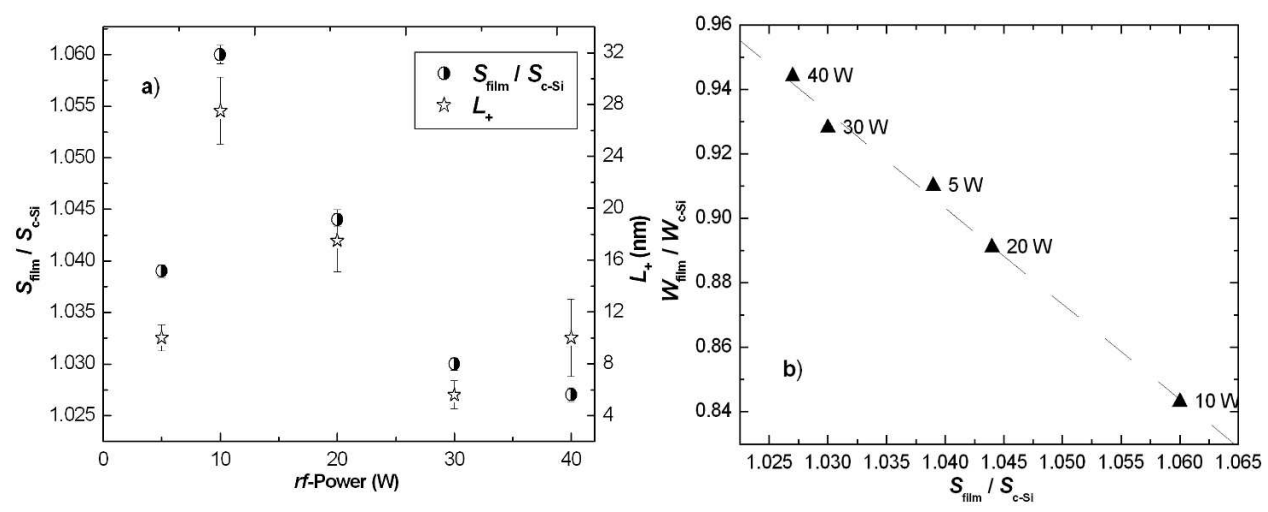

Fig. 1. (a) Normalized $S_{\text {film }}$ and positron diffusion length values (b) $S-W$ plot, for films deposited at different rf powers.

$\approx 1.027$ and $\approx 1.06$. From the literature, in a-Si:H four defect types which may trap positrons can be considered $[7,8]$ : monovacancies, divacancies, small vacancy clusters (with $\approx 5 \mathrm{Si}$ atoms missing) and large vacancy clusters or microvoids with a diameter of 1-2 nm, where even positronium (Ps) can also be formed. Indeed, our maximum and minimum $S_{\text {film }}$ values might correspond to trapping in large vacancy clusters/microvoids and in small vacancy type defects (probably with an open volume space similar to a monovacancy or divacancy), respectively. The highest $S_{\text {film }}$ value may also include contributions from positron surface states and from Ps caged inside the cavities. The film grown at $10 \mathrm{~W}$ contains, predominantly, large vacancy clusters, whereas the film grown at $40 \mathrm{~W}$ - mainly small vacancy type defects. The presence of the large vacancy cluster in the films is responsible for 
the degradation of the optoelectronic properties of these amorphous semiconductor thin films [9].

Figure 2 shows the Raman spectra obtained (a) at the lowest power $(1.5 \mathrm{~mW})$ of the laser, in the region of the TO-like band $\left(300-600 \mathrm{~cm}^{-1}\right)$ for all the samples and (b) at different laser powers for one of the samples (the shape is similar for all of them). Due to the weak illumination intensity, the spectra are noisier than usual. However, for the present study the important findings are the changes that are observed in these spectral bands for the films grown at different rf powers and after light soaking produced by the laser beam itself. All spectra exhibit a typical broad shape, as common in the Raman spectra of a-Si:H and of a-Si, with a maximum at around $480 \mathrm{~cm}^{-1}$, known as the TO-like band.

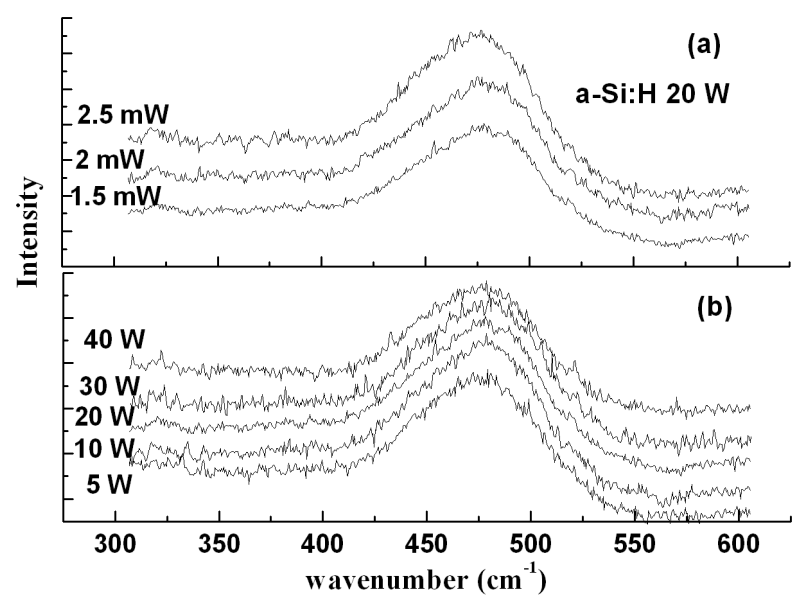

Fig. 2. The Raman spectra (a) for all films using laser power of $1.5 \mathrm{~mW}$, and (b) for the film deposited at $20 \mathrm{~W}$ measured at laser power of $1.5,2.0$ and $2.5 \mathrm{~mW}$, respectively (the vertical shifts are introduced for better visualization).

Figure 3 shows the values calculated from the Raman spectra, obtained with different laser power for different films. As can be seen in Fig. 3a, at the lowest laser power $(1.5 \mathrm{~mW})$, the $\Gamma_{\mathrm{TO}}$ value reaches its minimum for the film deposited at $P_{\mathrm{D}}=10 \mathrm{~W}$ that corresponds to the maximum value of $S_{\text {film }}$ of 1.06 , which can be understood as a strain stress relaxation process related to the formation of large vacancy cluster defects.

In Fig. 3b we can also see the structural differences induced by light soaking (i.e., with increasing the laser power). These differences are not related to thermal effects induced by laser irradiation, as proven by Stokes-anti-Stokes measurements performed on the c-Si sample, where no significant variation was detected. Our results agree quite well with the earlier referred theoretical model [4] where light soaking induces a decrease in the short-range order. In fact, when the laser power increases, the $\omega_{\mathrm{TO}}$ value decreases and this change is accompanied by an increase 

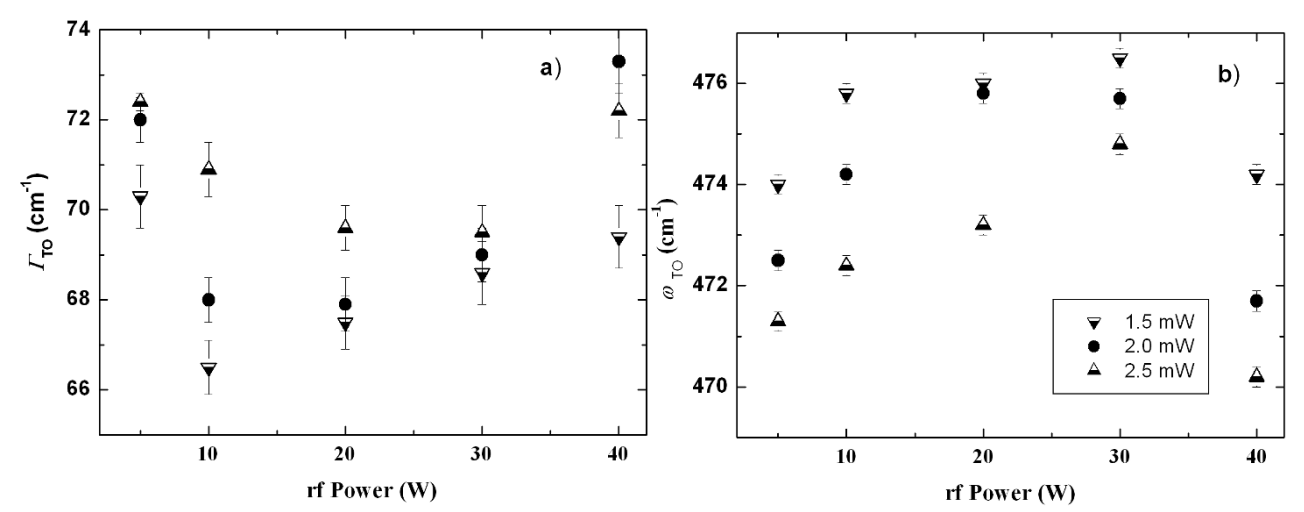

Fig. 3. Raman scattering spectroscopy results for successive measurements using increasing laser power on samples under different $\mathrm{rf}$ powers, (a) full width half maximum, $\Gamma_{\mathrm{TO}}$, and (b) the frequency of the peak position of the TO spectral band, $\omega_{\mathrm{TO}}$.

in the $\Gamma_{\mathrm{TO}}$ value, as can be seen in Figs. 3a and b. It is well established that the value of $\Gamma_{\mathrm{TO}}$ represents a good measure of the root mean square of the network bond angle distribution and the shift of $\omega_{\text {TO }}$ to lower wave numbers is associated with the increase in the average bond length [10]. Also, the use of positron annihilation spectroscopy in a-Si:H [7] has shown that light soaking does not change significantly the $S$ parameter of the film (there is only a slight increase), but the dominant change is observed in the $W$ parameter values. This means that the light soaking does not change the structure of the defects but only their chemical environment.

\section{Conclusions}

Slow positron beam Doppler broadening and micro-Raman spectroscopy measurements were made on a-Si:H films produced by PECVD at different $\mathrm{rf}$ powers to obtain information on the defect structure and on the degree of disorder. Positron annihilation spectroscopy shows clear differences in the structure of defects in films, small vacancy type defects and large vacancy clusters being the main defects present in the a-Si:H films. The Raman spectroscopy shows a decrease in structural disorder related to the presence of large vacancy clusters and this is associated with structural relaxation. The changes induced by light soaking, as observed in this work, are associated with atomic rearrangements, which are reflected as a decrease in short-range order.

\section{Acknowledgments}

The authors acknowledge Prof. A. Amorim da Costa and his collaborators for the use of the Raman setup and Dr. Alexander Kharlamov for help in 
the alignment of the Raman system. The support from the bilateral HungarianPortuguese Scientific exchange projects $\mathrm{P}-23 / 03$ and $\mathrm{P}-23 / 05$ was essential and is highly acknowledged.

\section{References}

[1] J.R. Abelson, Appl. Phys. A 56, 493 (1993).

[2] Y. Hiroyama, R. Suzuki, Y. Hirano, F. Sato, T. Motooka, Jpn. J. Appl. Phys. 34, 5515 (1995).

[3] D. Beeman, T. Tsu, M.F. Thorpe, Phys. Rev. B 36, 874 (1985).

[4] R. Biswas, Y.P. Li, Phys. Rev. Lett. 82, 2512 (1999).

[5] A.P. de Lima, C. Lopes Gil, P.M. Gordo, M. Duarte Naia, in: Workshop on Ion and Slow Positron Beam Utilization, OECD/NEA, Costa da Caparica (Portugal) 1998, p. 121.

[6] A. van Veen, H. Schut, J. Haakvoort, R.A.Vries, M.R. Ijpma, AIP Conf. Proc. 218, 171 (1990).

[7] X. Zou, Y.C. Chan, D.P. Webb, Y.W. Lam, Y.F. Hu, C.B. Beling, S. Fung, H.M. Weng, Phys. Rev. Lett. 84, 769 (2000).

[8] G. Amarendra, Defects Diff. Semicond. IV 200-202, 189 (2002).

[9] P.M. Gordo, M.F. Ferreira Marques, A.P. de Lima, G. Lavareda, C. Nunes de Carvalho, A. Amaral, Zs. Kajcsos, Rad. Phys. Chemistry 76, 220 (2007).

[10] M. Marinov, N. Zotov, Phys. Rev. B 55, 2938 (1997). 